Abstract-The golden king crab (Lithodes aequispinus) is a commercially important species in Alaska waters with an asynchronous reproductive cycle and lecithotrophic larvae. In this study, we qualitatively and quantitatively describe embryo development for this species. Six female multiparous golden king crab were captured from the Aleutian Islands, Alaska, and mated in the laboratory. Their embryos were photographed on average once every 9 days throughout embryogenesis. We describe 13 stages of embryo development on the basis of both visual observations and embryo morphometrics from 1241 measured embryos. Embryo development was similar to that of other cold-water crab species, with the exceptions that 1) golden king crab did not have a diapause and 2) that the average percentage of the area occupied by the yolk at hatching, at about $40 \%$, was much higher than that of other species. Both of these differences likely are attributable to the fact that the larvae are lecithotrophic and, therefore, do not need to synchronize hatch time with planktonic food availability but do need energy reserves to develop to the first crab stage. This study increases our understanding of the reproductive biology of the golden king crab and provides a baseline for future studies of embryo development.

Manuscript submitted 3 February 2015. Manuscript accepted 5 November 2015. Fish. Bull. 114:67-76 (2016).

Online publication date: 25 November 2015 . doi: 10.7755/FB.114.1.6

The views and opinions expressed or implied in this article are those of the author (or authors) and do not necessarily reflect the position of the National Marine Fisheries Service, NOAA.

\title{
Embryo development in golden king crab (Lithodes aequispinus)
}

\author{
W. Christopher Long (contact author) \\ Scott B. Van Sant \\ Email address for contact author: chris.long@noaa.gov \\ Resource Assessment and Conservation Engineering Division \\ Kodiak Laboratory \\ Alaska Fisheries Science Center \\ National Marine Fisheries Service, NOAA \\ 301 Research Court \\ Kodiak, Alaska 99615
}

The golden king crab (Lithodes aequispinus) is an important fishery species in Alaska waters; the annual harvest of the Aleutian Islands stock has averaged around 5-6 million lb since 1996 (NPFMC, 2015). In North American waters, golden king crab are distributed primarily along the upper portion of the continental slope, on seamounts, and in fjords in the Gulf of Alaska and to southern British Columbia, along the Aleutian Islands, and in the Bering Sea (Butler and Hart, 1962; Sloan, 1985; Donaldson and Byersdorfer, 2005). They have been harvested in the Bering Sea since the early 1980s, and the harvest levels there have been among the most stable for any crab species or stock (Orensanz et al., 1998; NPFMC, 2015).

Female golden king crab reach maturity, on average, at a carapace length (CL) of about 98-111 mm, depending on the stock and latitude (Jewett et al., 1985; Somerton and Otto, 1986). The reproductive cycle of golden king crab is not an annual event and is probably asynchronous in many areas (Hiramoto, 1985; Otto and Cummiskey, 1985; Somerton and Otto, 1986; Paul and Paul, 2000, 2001). Like all lithodids, female golden king crab must molt before mating (Paul and Paul, 2001), after which they extrude a clutch of up to 27,000 eggs (Jewett et al., 1985). Golden king crab have lower fecundities than similar-size red (Paralithodes camtschaticus) and blue (Paralithodes platypus) king crabs (Haynes, 1968; Somerton and MacIntosh, 1985; Swiney et al., 2012). They have much larger embryos because their larvae are lecithotrophic and therefore are supplied with greater energy reserves than are the larvae of red and king crab (Shirley and Zhou, 1997; Paul and Paul, 1999). For golden king crab, the brooding duration is about 362 days, and the time between the completion of larval hatching and extrusion of a new clutch is about 194 days; however, these parameters have been estimated only in the laboratory at temperatures that were higher than the temperatures experienced by crab in the field and are likely underestimates (Paul and Paul, 2001). After hatching, larvae pass through 3 zoeal stages and 1 glaucothoe stage before they molt to the first benthic crab stage (Shirley and Zhou, 1997; Paul and Paul, 1999).

One of the current gaps in our understanding of the biology of the golden king crab is the process of embryo development. The embryos of both the red king crab (Nakani- 
shi, 1987) and the blue king crab (Stevens, 2006) have been described in detail, but little attention has been given to the golden king crab up to this point. It is likely that, given the asynchronous reproductive cycle and the lecithotrophic development of larvae, golden king crab embryos are substantially different from those of the other 2 species. The asynchronous cycle could lead to greater differences among females, and lecithotrophic larval development could result in differences in embryo morphology, particularly in yolk size, at hatching. In this study, we examined and measured the embryos of golden king crab from time of extrusion though hatching and describe their development.

\section{Materials and methods}

Golden king crab were caught in commercial pots along the Aleutian Islands, Alaska, in the fall of 2005 and 2006 and transported to the Kodiak Laboratory of the NOAA Alaska Fisheries Science Center in air cargo. Crab were identified according to the methods of Donaldson and Byersdorfer (2005). Crab were held in 2000-L tanks with flow-through seawater chilled to $3-4^{\circ} \mathrm{C}$, a range that reflects the temperatures experienced by female golden king crab in the Aleutian Islands (Blau et al., 1996; Stabeno et al., 2005) and were fed chopped frozen fish and squid to excess. Tanks were covered with opaque sheets of foam both to provide insulation and to keep the females in mostly dark conditions.

Six females, 4 captured in 2005 and 2 caught in 2006, were used in this study. Females were either late-stage ovigerous, as evinced by eyed embryos, or they were hatched out, as evinced by empty egg cases found when collected. They were between 120 and 141 mm CL. Six mature males, 128-132 mm CL, were kept with pre-mating females in a holding tank because females may molt and mate anywhere from 5 to 464 days after they finish hatching larvae (Paul and Paul, 2001).

When pre-mating grasping occurred, a crab pair was moved to a separate tank to ensure that the female would not be eaten after molting. After the females molted, they mated and extruded a new clutch of eggs. At this point, they were placed in another tank where all the postmating females were held together with no males. The day of extrusion was considered day 1 of brooding and embryo development.

Embryos were collected regularly throughout development from a random location within the clutch. Throughout development for each female, time between samples varied with the stage of development but averaged once every 9 days or a mean of 48 times (range: 42-54 times). At sampling, embryos were examined and photographed under a stereomicroscope. Uneyed embryos were stained for 5 min in Bouin's solution to enable staging (Stevens, 2006). In addition, for image analysis, photographs were taken of up to 10 unstained embryos at $90^{\circ}$ to the sagittal plane under a stereomicroscope. These images were calibrated each day with a micrometer because the scope had an adjustable zoom and this calibration ensured that measurements were accurate. A total of 1241 embryos were measured. On a number of occasions, the calibration procedure was not followed, and we did not perform image analysis on the photographs, although stage data were still collected and used. Measurements were made with Image-Pro Plus ${ }^{1}$, vers. 7.0 (Media Cybernetics Inc., Rockville, MD).

In this study, the term egg refers to the entire embryo (i.e., the entire contents of the fertilized egg), the term embryo refers to the differentiated part of the egg as distinct from the yolk, and the term yolk refers to the undifferentiated deutoplasm. For each egg, the area and the mean, minimum, and maximum diameter were measured. The mean, minimum, and maximum diameters were determined from 180 measurements of the diameter at $2^{\circ}$-intervals around the entire egg. When the embryo became visible, the yolk area also was measured by tracing the yolk in Image-Pro Plus, and the percentage of egg area that was yolk (hereafter percent area of yolk, calculated as yolk area $\times 100 /$ egg area; before the embryo becomes visible, the yolk area equals the egg area) and the embryo area (calculated as egg area - yolk area) were determined. Finally, when the eyes became visible, the eye area and the mean, minimum, and maximum diameter were measured with same techniques as above. All measurements were made at $90^{\circ}$ to the sagittal plane.

Stages were described by visual examination of the eggs as well as on the basis of the changes observed in the measured parameters, and these descriptions were based, in part, on staging systems previously developed for embryos of the blue king crab (Stevens, 2006) and snow crab (Chionoecetes opilio) (Moriyasu and Lanteigne, 1998). The median stage of development (see the Results section for descriptions) was determined for each female on each sampling day. The data for developmental stages were fitted to a model of multiple-transitions between stages (MT model, Long, 2016). In brief, each stage transition was modeled as a logistic regression:

$$
\text { stage }=\frac{1}{1+\left(\frac{t}{t_{50}}\right)^{s}},
$$

where $t=$ the time in days;

$t_{50}=$ the time at which half of the embryos have transitioned to the next stage; and

$s=$ a parameter that is proportional to the slope at the transition.

The data were fitted by using maximum like-lihood in R, vers. 2.14.0 (R Development Core Team., 2011).

Morphometric analysis was used to visualize differences among the developmental stages and to examine whether embryo measurements can be used to distin-

\footnotetext{
${ }^{1}$ Mention of trade names or commercial companies is for identification purposes only and does not imply endorsement by the National Marine Fisheries Service, NOAA.
} 
guish the different stages. To visualize stage differences, the morphometric data were normalized and then analyzed with principal component analysis (PCA). To determine whether morphometric measurements would allow us to distinguish the stages, the data were fourth-root transformed and an analysis of similarity (ANOSIM) was performed on a Bray-Curtis similarity matrix with female and stage as factors. All morphometric analyses were performed in Primer, vers. 6.1.15 (PRIMER-E Ltd., Ivybridge, U.K.; Clarke and Warwick, 2001).

When females approached time of hatching, they were isolated in individual containers with flowthrough water. Larvae released from each female were collected in nets that were checked daily. The first and last day of hatching were noted.

\section{Results}

Females extruded their eggs between 25 September 2006, and 6 May 2007, and they began hatching between 3 December 2007, and 23 July 2008. The mean duration of brooding (from extrusion to the first day of hatching) was 437.6 days (standard deviation [SD] 6.7). We identified 13 stages of embryo development (12 of them are shown in Fig. 1) by visual examination of the embryos and their morphometric features. The MT model was an excellent fit to the data for stage transitions (Fig. 2) and provided estimates for the average day of transition $\left(t_{50}\right)$ for each stage (Table 1$)$. The mean duration of stages was between 10 and 95 days. There were some differences among the females (Fig. 2 ), as demonstrated by the increased SD in the average embryo stage among females, particularly during some of the shorter stages (i.e., stages 3, 4, and 5) and during stages 9,10 , and 11 . The following sections describe the 13 stages identified.

\section{Stage 0 (precleavage)}

At this stage, the eggs were newly extruded and firmly attached to the pleopods; however, no division had yet occurred. There was no visible separation between the yolk, which was a creamy yellow, and the egg membrane.

\section{Stage 1 (cleavage and blastula)}

This stage began with the first cleavage to the 2-cell stage; this cleavage occurred between days 10 and 11 , and development progressed rapidly (Fig. 1A). By day 13 , most yolks were at the 8 -to 16 -cell stage and by day 15 they were at the 32-to 64-cell stage. During these stages of early division, the yolk often took on a lumpy shape and was frequently separated from the egg membrane, and a nucleus was visible in each cell as a lightcolored circle when stained with Bouin's solution. By about day 50, the blastula formed, and individual cells were no longer discernible.

\section{Stage 2 (gastrula)}

The beginning of this stage was marked by the blastopore (Fig. 1B) becoming visible in embryos stained with Bouin's solution. This stage began on average on day 60 and lasted for about 32 days (Table 1 ).

\section{Stage 3 (v-shaped embryo)}

This stage began with the appearance of the embryo as a yellow v-shape when it was stained in Bouin's solution (Fig. 1C). This stage was short, beginning on about day 91 and lasting for about 24 days (Table 1).

\section{Stage 4 (prenauplius)}

During this stage, the embryos began to rapidly form differentiated parts. This stage began when the optical lobe, antennules, antennas, mandibles, and abdomen were visible as separate entities as opposed to the undifferentiated v-shape of the previous stage in embryos stained with Bouin's solution (Fig. 1D). The individual parts were still somewhat indistinct and widely separated from each other; in particular, the abdomen was well-separated from the optical lobe, mandibles, and antennas. This stage lasted for approximately 27 days, starting on day 115 (Table 1).

\section{Stage 5 (nauplius)}

This stage was brief, starting at around day 142 and lasting only about 18 days (Table 1). During this stage, the optical lobes, antennules, antennas, mandibles, and abdomen were more distinct, and they lengthened and coalesced so that the abdomen was situated between the antennas and mandibles and immediately below the optical lobes (Fig. 1E).

\section{Stage 6 (maxilliped formation)}

This stage was another brief one, starting at around day 160 and lasting only about 15 days (Table 1). It began when the maxillipeds became distinct from the mandibles. During this stage, the abdomen became more elongate (Fig. 1F). Before this stage, there were almost no changes in the morphological features of the eggs; the egg size and dimensions remained constant, and the embryo was not visible in unstained embryos (Fig. 3). But, during this stage, the embryo became visible in unstained embryos, and size and percent area of yolk began to decrease (Fig. 4).

\section{Stage 7 (metanauplius)}

The metanauplius stage began when the abdomen was fully bifurcated and the carapace became visible. This stage was characterized by the lengthening of the antennules, antennas, mandibles, and maxillipeds (Fig. 1G). Late in this stage, the telson became visible. Growth of the embryo and reduction in the yolk hap- 

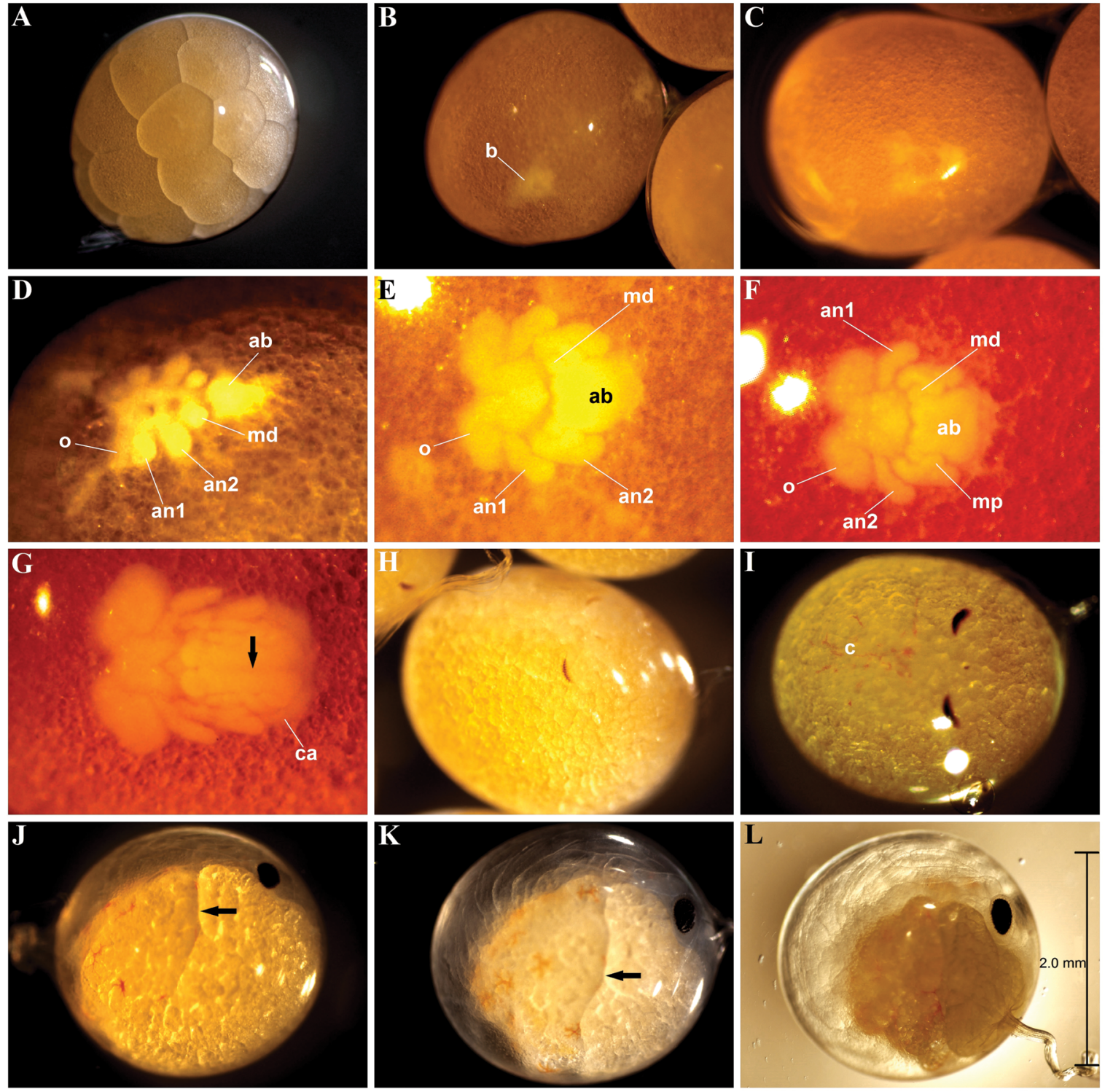

Figure 1

Photographs of golden king crab (Lithodes aequispinus) embryos taken throughout development. Sections A-L show embryo stages 1-12, respectively; stage 0 is not provided. In sections B-G, photographs are of stained embryos. Light levels and colors in photographs have been adjusted to make the pertinent details clear. Key morphological features used to identify stages are labeled: blastopore (b), optical lobe (o), antennule (an1), antenna (an2), abdomen (ab), mandible (md), maxilliped ( $\mathrm{mp}$ ), carapace (ca), and chromatophores (c). In section $\mathrm{G}$, the arrow indicates bifurcated abdomen. In panel $\mathrm{J}$, the arrow indicates partially bifurcated yolk. In section $\mathrm{K}$, the arrow indicates a fully bifurcated yolk.

pened at a very slow rate (Figs. 3 and 4). This stage started at around day 175 and lasted 38 days.

\section{Stage 8 (eye formation)}

The beginning of this stage, which began on day 213 , was marked by the eyes of the embryo becoming visible as thin, pigmented crescents in unstained embryos
(Fig. 1H). During this stage, the size of the eyes rapidly increased-the width (minimum diameter) increasing faster than the length (maximum diameter)—so that the shape gradually became a fuller crescent shape (Fig. 5). Although embryo growth was faster in this stage than in stage 7 , it was still relatively slow (Fig. 3 ). The telson became distinct and developed setae in embryos as evident in embryos stained with Bouin's solution. This stage 


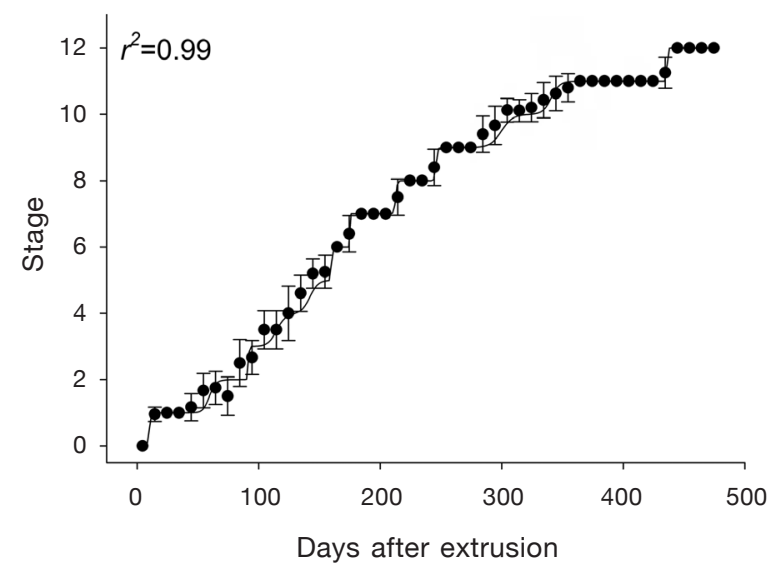

Figure 2

Average (among females) developmental stage of golden king crab (Lithodes aequispinus) embryos plotted against days from extrusion, for each 10-day increment of embryo development. The line represents the best fitting multiple-stage transition model. Error bars indicate \pm 1 standard deviation of the mean. See Table 1 for parameter estimates. $r^{2}=$ coeficcient of determination.

marked the midpoint in embryo development, beginning on day 213 and lasting for about 33 days (Table 1).

\section{Stage 9 (chromatophore formation)}

The beginning of this stage was marked by chromatophores becoming visible in unstained embryos (Fig. 1I). Chromatophores increased in size and num- ber throughout this stage. Growth of the embryo and shrinking of the yolk accelerated from the previous stage (Figs. 3 and 4). The eyes grew rapidly, both in length and width. Because width increased much faster than length, the eyes progressed from a crescent shape to a nearly spherical one (Fig. 5B). From this stage onward in development, egg area began to increase as well (Fig. 3), although during this stage the increase was slight. The yolk became separated into 2 halves when the egg was viewed dorsally. This stage began on day 246 and lasted for 54 days.

\section{Stage 10 (rapid growth)}

This stage, which lasted about 41 days and began on day 300 (Table 1), was marked by a rapid increase in the size of the egg, embryo, and eyes, and a slight decrease in the yolk area and percent area of yolk (Figs. 3,4 , and 5 ). The shape of the eye did not change during this period and remained nearly spherical (Fig. 5B). When viewed on the sagittal plane, the yolk developed a partial anterior-posterior bifurcation (Fig. 1J). We marked the beginning of this stage with the beginning of this bifurcation and marked the completion of this stage with the completion of this bifurcation.

\section{Stage 11 (prehatching)}

At the beginning of this stage, the yolk was completely bifurcated (Fig. 1K). Growth of the eye and embryo were at their maximum rates during this stage (Figs. 3 and 5). Yolk area and percent area of yolk dropped rapidly, with the percent area of yolk dropping from $70 \%$ to less than $50 \%$ (Fig. 4). The eyes continued to lengthen and increase in area, but the width did not

Table 1

Timing and duration of each stage of embryo development of golden king crab (Lithodes aequispinus). Mean values, with standard errors in parentheses, are provided for $t_{50}$ (the time in days at which $50 \%$ of the embryos transitioned to a given stage), and for $s$, a slope parameter for the transition to the next stage. Length is the average length (in days) of each stage, and $-s / t_{50}$ is a measure of the rapidity of the transition to the next stage.

\begin{tabular}{|c|c|c|c|c|c|}
\hline Stage & Description & $t_{50}(\mathrm{~d})$ & Length (d) & $s$ & $-s / t_{50}$ \\
\hline 0 & Precleavage & - & 10.00 & $-509.76(\mathrm{SE} 1660.70)$ & 50.98 \\
\hline 1 & Cleavage and blastula & $10.00($ SE 0.03$)$ & 49.47 & $-22.76($ SE 0.37$)$ & 0.38 \\
\hline 2 & Gastrula & 59.47 (SE 0.20) & 32.02 & $-278.22(\mathrm{SE} 71.06)$ & 3.04 \\
\hline 3 & V-shaped embryo & 91.49 (SE 0.16) & 23.53 & $-31.24(\mathrm{SE} 3.74)$ & 0.27 \\
\hline 4 & Prenauplius & $115.02(\mathrm{SE} 1.35)$ & 27.18 & -42.08 (SE 2.39) & 0.30 \\
\hline 5 & Nauplius & $142.20(\mathrm{SE} 0.73)$ & 17.75 & $-577.71(\mathrm{SE} 233.35)$ & 3.61 \\
\hline 6 & Maxilliped formation & $159.95($ SE 0.80$)$ & 14.58 & $-2006.63($ SE 8853.47) & 11.50 \\
\hline 7 & Metanauplius & $174.53(\mathrm{SE} 6.47)$ & 38.18 & $-314.76(\mathrm{SE} 27.35)$ & 1.48 \\
\hline 8 & Eye formation & $212.71(\mathrm{SE} 0.19)$ & 33.32 & $-420.90($ SE 67.60$)$ & 1.71 \\
\hline 9 & Chromatophore formation & $246.03(\mathrm{SE} 0.25)$ & 53.79 & $-65.52(\mathrm{SE} 0.37)$ & 0.22 \\
\hline 10 & Rapid growth & $299.82(\mathrm{SE} 0.39)$ & 40.96 & -99.09 (SE 0.05) & 0.29 \\
\hline 11 & Prehatching & 340.78 (SE 0.29) & 95.25 & -1266.33 (SE 36.33) & 2.90 \\
\hline 12 & Hatching & $436.03($ SE 0.08$)$ & 25.67 & - & - \\
\hline
\end{tabular}



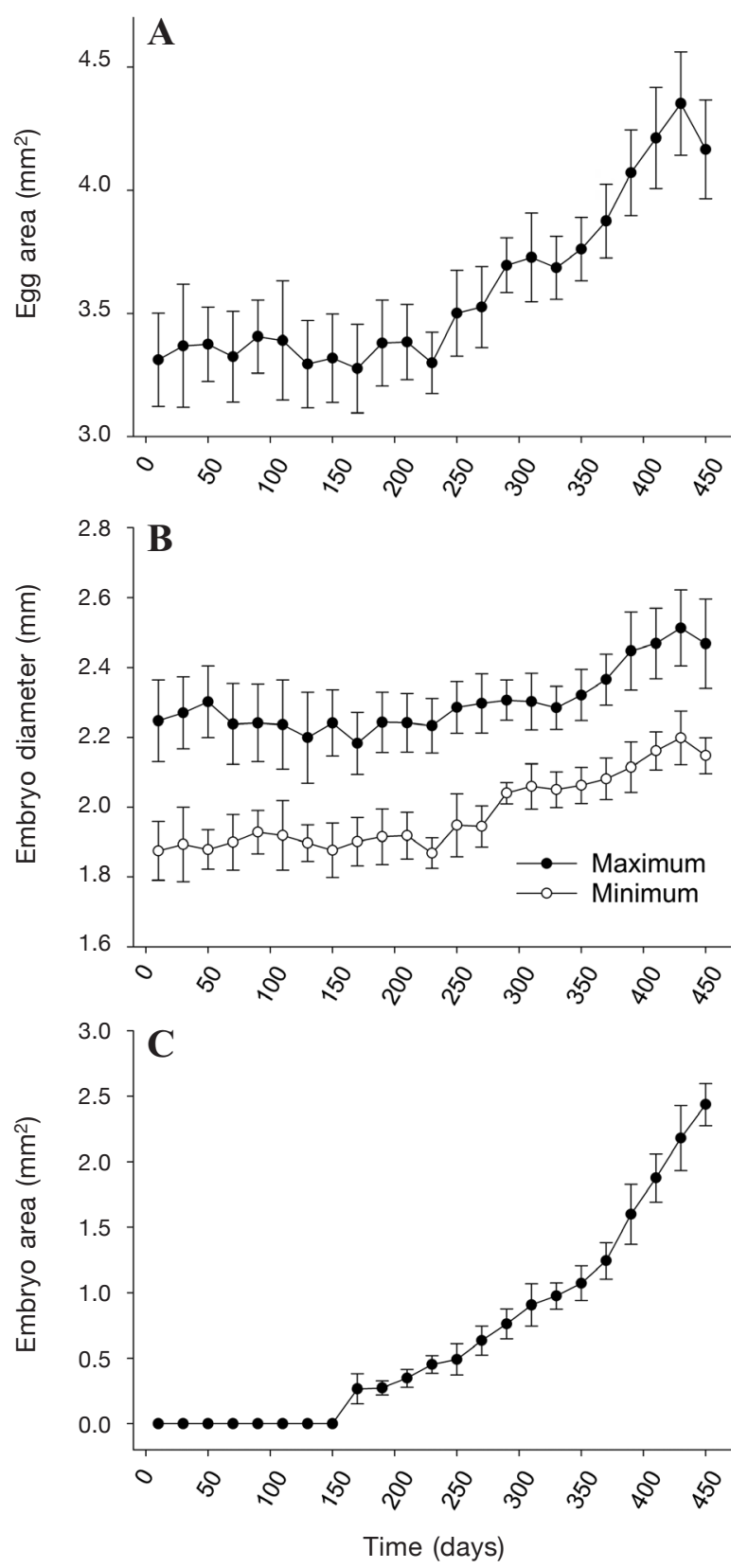

Figure 3

Average (A) egg area, (B) egg diameter, and (C) embryo area for all embryos measured during each 20 -day increment throughout larval development of golden king crab (Lithodes aequispinus). Error bars indicate \pm 1 standard deviation of the mean.

change, and, as a result, the eye became more oblong in shape (Figs. 1K and 5B). Additionally, development of the ommatidia gave the eye a visibly granular texture and caused a halo effect around the eye. This stage, beginning on day 340, was the longest one, lasting 95 days on average (Table 1 ). The end of this stage was marked by the beginning of hatching.

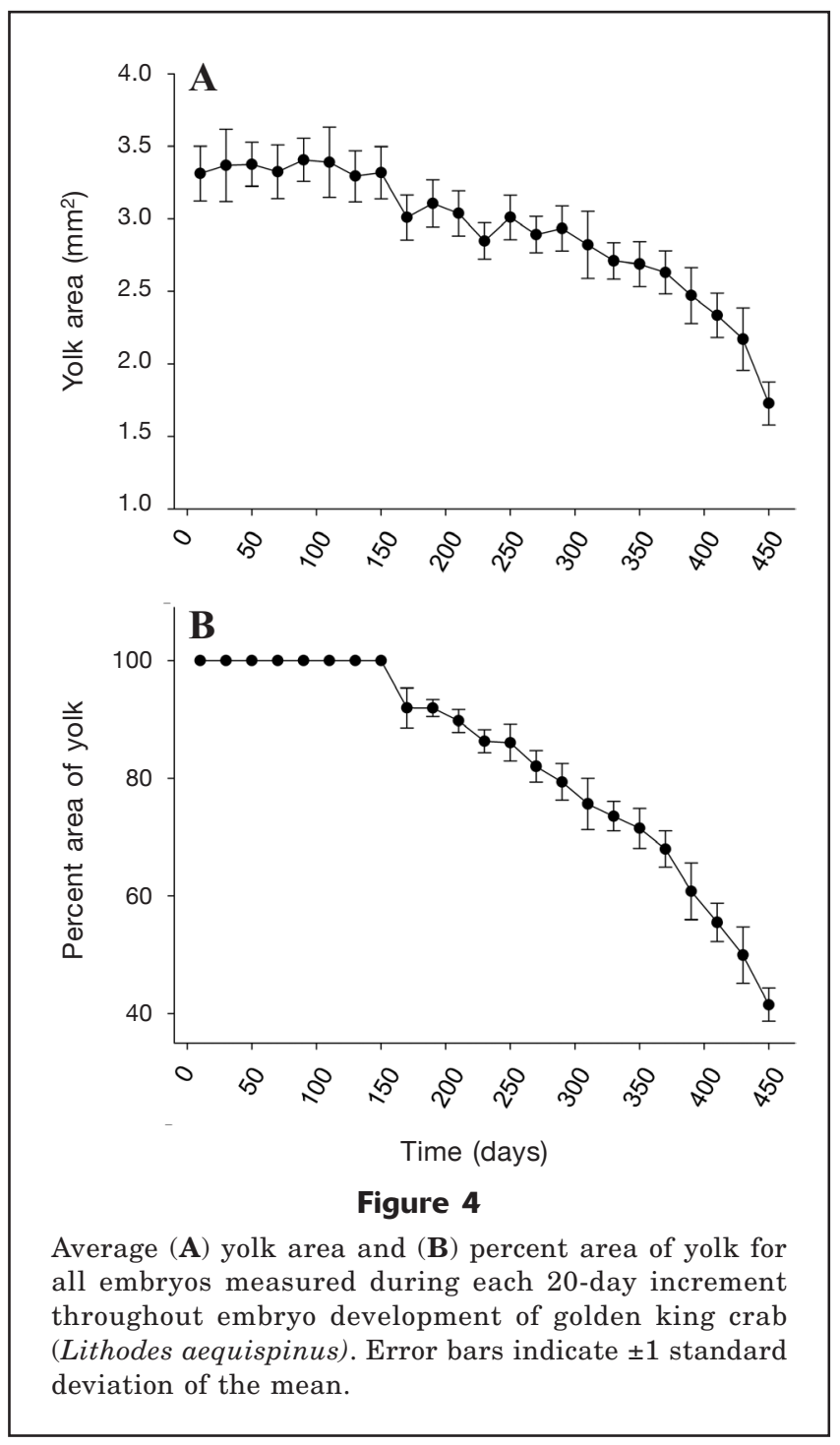

\section{Stage 12 (hatching)}

During hatching, embryos reached their maximum size, and the yolks reached their minimum, at about $40 \%$ of the total area of the egg (Figs. 1L, 3, and 4). The anterior and posterior sections of the yolk became differentiated from each other, with the posterior becoming more globular and less distinct (Fig. 1L). Hatching began on about day 436, and the last day of hatching occurred on average at 464 days (SD 8.6), and the average duration of hatching was 25.7 days (SD 6.1) (Table 1).

\section{Morphometric analysis}

The first 2 principal components (PCs) of the PCA explained $83.3 \%$ and $9.5 \%$ of the variance in embryo morphometrics of golden king crab and were retained (Table 2). The first PC was positively correlated with yolk size, negatively correlated with embryo, eye, and egg size, and was interpreted as embryo maturity, and 

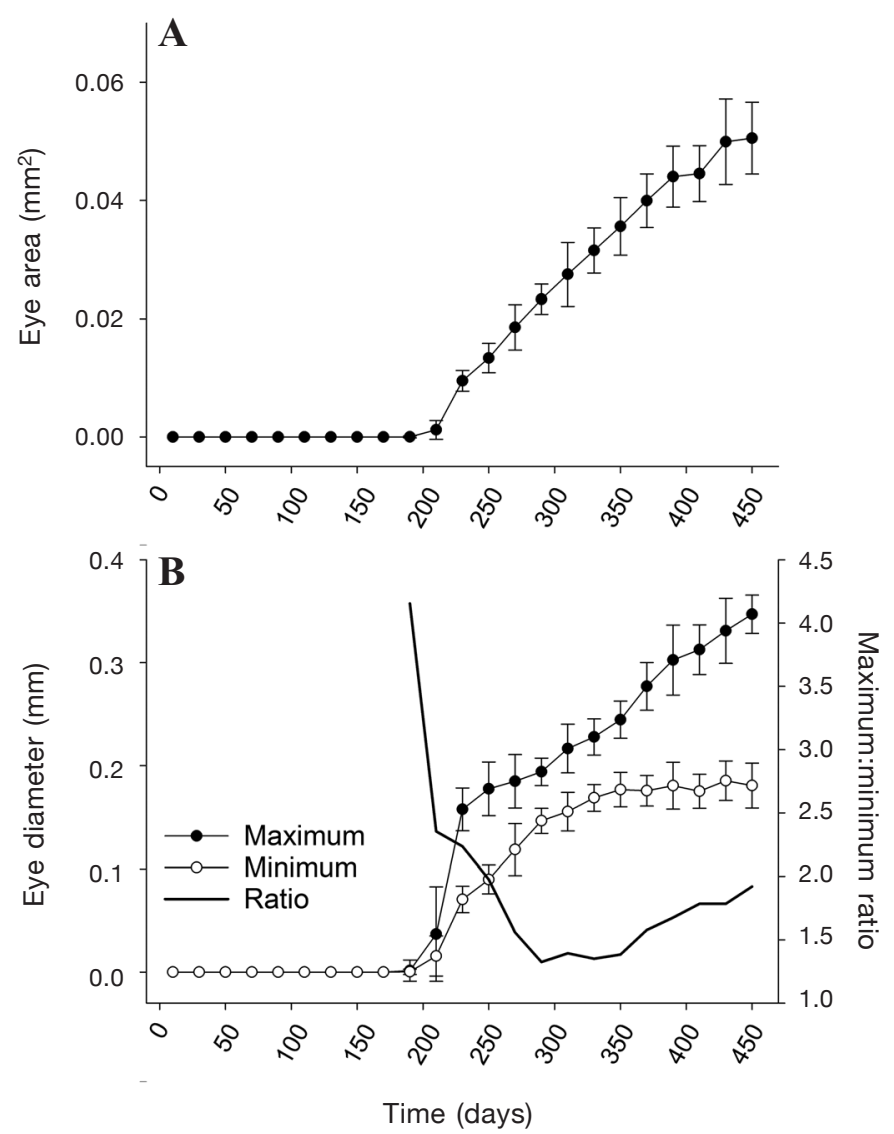

Figure 5

Average (A) eye area and (B) maximum and minimum eye diameters for all embryos measured during each 20-day increment during embryo development of golden king crab (Lithodes aequispinus). In section $\mathrm{B}$, the line without points represents the ratio between the maximum and minimum eye diameters as an indicator of eye shape. Error bars indicate \pm 1 standard deviation of the mean.

smaller numbers corresponded to more mature embryos (Table 2, Fig. 6). The second PC was negatively correlated with egg size and yolk area and explained variation in egg sizes within a given stage (Table 2, Fig. 6). There were significant differences in embryo morphometrics among females (ANOSIM: global $R=0.229, P<0.001$ ) and stages (ANOSIM: global $R=0.730, P<0.001)$. Although it is significant, the low value for the global $R$ statistic indicates that, although there were statistical differences among the females the differences were probably negligible (Clarke and Warwick, 2001). Ordination by both PCA and nonmetric multidimensional scaling confirmed this conclusion. Pairwise comparisons of the stages showed that, in general, stages $0-5$ could not be distinguished from each other (i.e., $P>0.050$ or global $R<0.200$ and usually both) and that stages 6-12 each differed significantly from all other stages (in all cases $P<0.001$ and global $R>0.300)$. These results confirmed the results from PCA (see the PCA plot, Fig. 6).

\section{Discussion}

Embryo development in golden king crab examined in this study was similar to that reported in other studies for the red king crab (Nakanishi, 1987), blue king crab (Stevens, 2006), and snow crab (Moriyasu and Lanteigne, 1998). Most of the stages described in our study match closely stages identified in those other studies. This similarity is not surprising, especially for the similarities noted among the 3 closely related species of king crabs. Despite the general similarity, there were some aspects of embryogenesis in golden king crab that were distinct, such as the lack of a diapause and the rate of decrease in yolk area.

Blue king crab undergo a diapause stage that lasts for approximately 2 months (Stevens, 2006) between the stages of chromatophore formation and eye enlargement (approximately equivalent to our stages 9 and 10). Snow crab undergo a 6 -month diapause once they reach the gastrula stage and a second diapause of 3-4 months after the eye pigment formation stage (approximately equivalent to our stage 8 ) in the field (Moriyasu and Lanteigne, 1998). Tanner crab (Chionoecetes bairdi) also undergo a diapause of 3-6 months after the gastrula stage (Swiney, 2008). In snow crab, the diapauses seem to be the mechanism for switching between a 1-year and 2-year brooding period (Moriyasu and Lanteigne, 1998; Webb et al., 2007), and, in both snow crab and Tanner crab, the purpose of varying the duration of embryonic development is likely to ensure that larval release coincides with the spring planktonic bloom (Swiney, 2008). It is probable that the golden king crab lacks a diapause stage because its larval development is lecithotrophic. Lecithotrophic larvae do not need to feed, and golden king crab larvae do not appear to feed at all (Shirley and Zhou, 1997); therefore, there is no need to synchronize the release of larvae with food availability and, thus, there is no advantage to having a diapause stage. This interpretation matches with the observation of an asynchronous reproductive cycle for this species (Somerton and Otto, 1986).

Another major, and expected, difference in golden king crab embryogenesis, compared with that of other crab species, is the rate at which the yolk area decreases, and the amount of yolk remaining at hatching. The percent area of yolk decreases at a much faster rate in blue king crab than it does in golden king crab, and at the beginning of hatching blue king crab have only about $13 \%$ area of yolk (Stevens, 2006). Snow crab embryos have, at most, a trace of yolk left at hatching (Moriyasu and Lanteigne, 1998), as do Tanner crab (Swiney et al., in press). In this study, golden king crab had more than $40 \%$ area of yolk remaining at hatching. Again, however, this difference is driven by the requirements of lecithotrophic larval development; the larvae 


\section{Table 2}

Results of a principal component analysis of embryo morphometrics of golden king crab (Lithodes aequispinus). Percent variation and cumulative variation represent the percentage of variance in the data explained by each principal component (PC) and the cumulative variance explained. $\mathrm{Max}=$ maximum; $\min =$ minimum.

\begin{tabular}{|c|c|c|c|}
\hline $\mathrm{PC}$ & Eigenvalues & Variation (\%) & $\begin{array}{c}\text { Cumulative } \\
\text { variation }(\%)\end{array}$ \\
\hline 1 & 9.160 & 83.3 & 83.3 \\
\hline 2 & 1.040 & 9.5 & 92.8 \\
\hline 3 & 0.521 & 4.7 & 97.5 \\
\hline 4 & 0.216 & 2.0 & 99.5 \\
\hline 5 & 0.024 & 0.2 & 99.7 \\
\hline 6 & 0.018 & 0.2 & 99.8 \\
\hline 7 & 0.015 & 0.1 & 100 \\
\hline 8 & 0.003 & 0 & 100 \\
\hline 9 & 0.000 & 0 & 100 \\
\hline 10 & 0.000 & 0 & 100 \\
\hline 11 & 0.000 & 0 & 100 \\
\hline \multirow{2}{*}{\multicolumn{2}{|c|}{ Measurement }} & \multicolumn{2}{|c|}{ Eigenvectors } \\
\hline & & PC1 & $\mathrm{PC} 2$ \\
\hline \multicolumn{2}{|c|}{ Egg area } & -0.301 & -0.385 \\
\hline \multicolumn{2}{|c|}{ Egg max diameter } & -0.217 & -0.614 \\
\hline \multicolumn{2}{|c|}{ Egg min diameter } & -0.278 & -0.098 \\
\hline \multicolumn{2}{|c|}{ Egg mean diameter } & -0.301 & -0.377 \\
\hline \multicolumn{2}{|c|}{ Embryo area } & -0.324 & 0.072 \\
\hline \multicolumn{2}{|c|}{ Yolk area } & 0.277 & -0.449 \\
\hline \multicolumn{2}{|c|}{ Percent area of yolk } & 0.324 & -0.122 \\
\hline \multicolumn{2}{|c|}{ Eye area } & -0.324 & 0.124 \\
\hline \multicolumn{2}{|c|}{ Eye max diameter } & -0.321 & 0.154 \\
\hline \multicolumn{2}{|c|}{ Eye min diameter } & -0.314 & 0.182 \\
\hline \multicolumn{2}{|c|}{ Eye mean diameter } & -0.319 & 0.172 \\
\hline
\end{tabular}

need a greater energy reserve at hatching than the energy reserve of feeding larvae to ensure that they can develop to the first crab stage.

The developmental time between extrusion and hatching (brooding time) and the duration of hatching in this study both differ from previous reported values. Paul and Paul (2001) reported an average brooding time of 362 days (2269 degree-days [number of days multiplied by the average temperature in degrees Celsius]) and an average hatching duration of 34 days (202 degree-days), whereas we observed an average brooding time of 436 days ( 1526 degreedays) and an average hatch duration of 26 days ( 91 degree-days).

This dissimilarity between studies may have resulted from the crabs in each study having come from different locations: Prince William Sound (Paul and Paul, 2001) and the Aleutian Islands (in this study). However, it is more likely due to the differences in holding temperatures. Paul and Paul (2001) held crab at the ambient temperatures found at a depth of 75 $\mathrm{m}$ in Prince William Sound, $3.5-9.5^{\circ} \mathrm{C}$, a range $1-4^{\circ} \mathrm{C}$ higher than the range of temperatures of the deeper waters in Prince William Sound where the golden king crab occur. We held the crab at a constant temperature between $3^{\circ} \mathrm{C}$ and $4^{\circ} \mathrm{C}-\mathrm{a}$ range that is probably more reflective of their natural environment. Along the Aleutian Islands, most mature females are distributed at depths between about 300 and $500 \mathrm{~m}$ (Blau et al., 1996) where temperatures vary from about 3.5 to 4.5 throughout the year (Stabeno et al., 2005). The increase in the number of degree-days necessary for development with increasing temperature also occurs in the blue king crab (Stevens et al., 2008) and snow crab (Webb et al., 2007).

Morphometric analysis of golden king crab embryos failed to provide a quantitative method for staging larvae. Stevens (2006) successfully used multivariate statistical techniques to identify stages in blue king crab and indicated that analyses could be used to better compare embryogenesis over a diverse range of crustacean species. Using similar techniques, we were not able to distinguish among the first 5 stages, which represent about 160 days or a third of the developmental time for embryos. These stages are difficult to distinguish morphometrically because the embryo and eyes are not yet measurable. This technique worked for the blue king crab because eggs decreased steadily in size during these stages (Stevens, 2006), but it cannot work for the golden king crab because the egg size in this species remained constant for about the first 250 days of development in our study. On the other hand, the MT model (Long, 2016) provided an excellent fit to the data for stage transitions, explaining $99 \%$ of the variation in the data. These data provide quantitative estimates of the average duration of each embryonic stage and will serve as a baseline for studies of embryo development in the golden king crab.

Climate change, that is, changes in temperature (Webb et al., 2007; Stevens et al., 2008), and ocean acidification (Long et al., 2013a; Long et al., 2013b) can have substantial effects on the early life histories of cold-water crabs. This study provides a baseline for future studies that examine either variability in embryo development times among different populations of the golden king crab or the effect of environmental variables on embryo development. Future studies on the reproductive biology of the golden king crab should determine whether primiparous and multiparous crabs differ in their reproductive cycles (e.g., Moriyasu and Lanteigne, 1998; Swiney, 2008; Swiney and Long, 2015) and what drives the high variability in the time between the end of hatching and the time of mating in the golden king crab (Paul and Paul, 2001).

\section{Acknowledgments}

We thank the observers in fishery observer program of Alaska Department of Fish and Game and the captains and crews of fishing vessels from which the golden king 


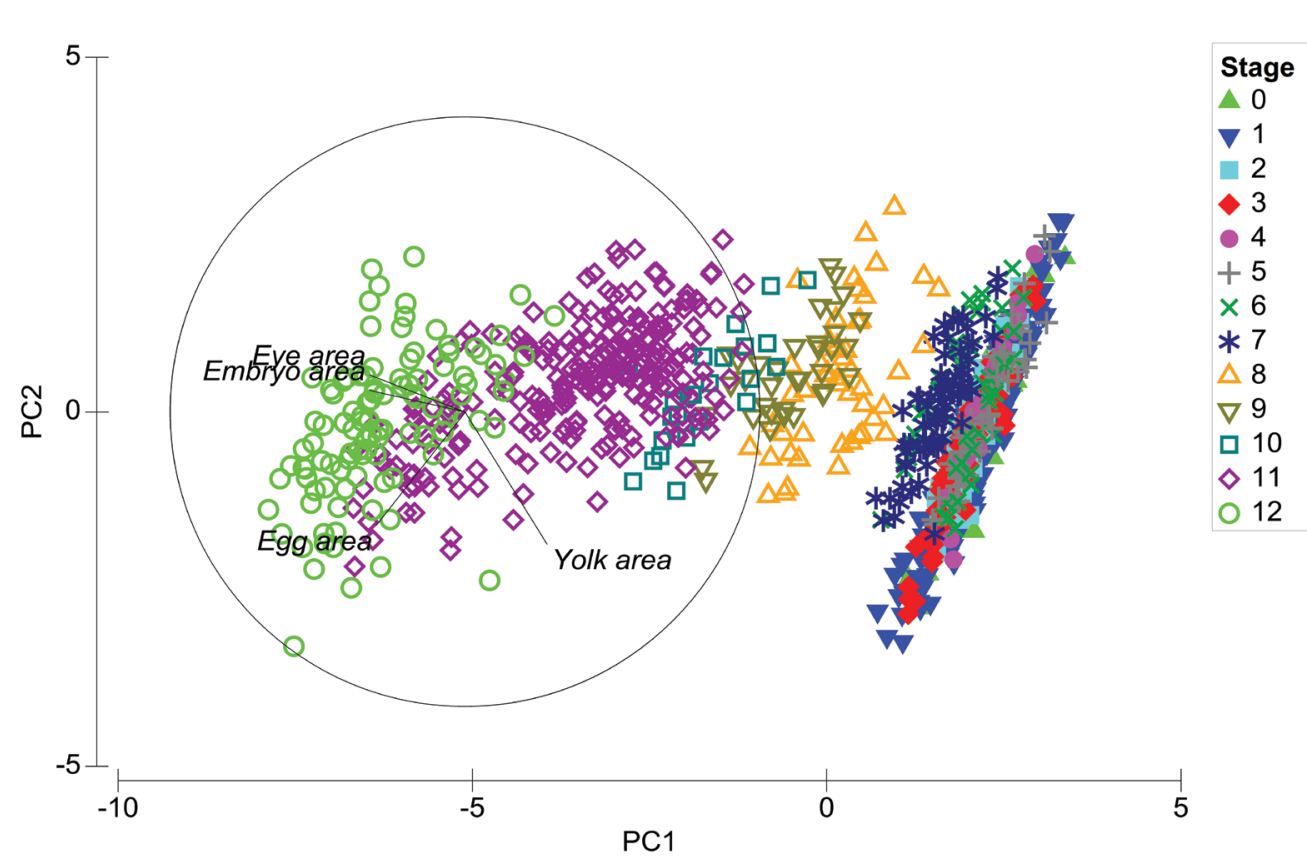

Figure 6

Plot of principal component analysis (PCA) of morphometrics of golden king crab (Lithodes aequispinus) embryos during 13 stages of development. Each point represents a single embryo. The first 2 PCs (plotted here ) accounted for $93 \%$ of the variance in the data. Vectors are shown for egg, embryo, yolk, and eye areas.

crab were collected for this experiment. We thank B. Stevens for training, sharing techniques, and helpful suggestions, K. Reppond for assistance; and R. Foy for discussions. We also thank the staff, interns, and volunteers of the seawater laboratory complex of the Kodiak Laboratory, NOAA Alaska Fisheries Science Center, for assistance in performing experiments. Previous versions of this article were improved by comments from R. Foy, J. Long, K. Swiney, and 3 anonymous reviewers.

The metadata associated with this project are available at InPort (website) under the catalog item ID 26894 and the title "AFSC/RACE/SAP/Long: Data from: Embryo development in golden king crab, Lithodes aequispinus." The data are available at the following website.

\section{Literature cited}

Blau, S. F., D. Pengilly, and D. A. Tracy.

1996. Distribution of golden king crabs by sex, size, and depth zones in the Eastern Aleutian Islands, Alaska. In High latitude crabs: biology, management, and economics. Alaska Sea Grant College Program Rep. AKSG-96-02, p. 167-185. Univ. Alaska Fairbanks, Fairbanks, AK.

Butler, T., and J. F. Hart.

1962. The occurrence of the king crab, Paralithodes camtschatica (Tilesius), and of Lithodes aequispina
Benedict in British Columbia. J. Fish. Res. Board Can. 19:401-408. Article

Clarke, K. R., and R. M. Warwick.

2001. Change in marine communities: an approach to statistical analysis and interpretation, $2^{\text {nd }} \mathrm{ed}, 172 \mathrm{p}$ PRIMER-E, Plymouth, U.K.

Donaldson, W. E., and S. C. Byersdorfer.

2005. Biological field techniques for lithodid crabs, $76 \mathrm{p}$. Univ. Alaska Fairbanks, Alaska Sea Grant College Program Rep. AK-SG-05-03.

Haynes, E.

1968. Relation of fecundity and egg length to carapace length in the king crab, Paralithodes camtschatica. Proc. Natl. Shellfisheries Assoc. 58:60-62.

Hiramoto, K.

1985. Overview of the golden king crab, Lithodes aequispi$n a$, fishery and its fisheries biology in the Pacific waters of Central Japan. In Proceedings of the International King Crab Symposium, Alaska Sea Grant College Program AK-SG-85-12; Anchorage, AK, 22-24 January, p. 297-317. Univ. Alaska Fairbanks, Fairbanks, AK.

Jewett, S. C., N. A. Sloan, and D. A. Somerton.

1985. Size at sexual maturity and fecundity of the fjorddwelling golden king crab Lithodes aequispina Benedict from northern British Columbia. J. Crust. Biol. 5:377-385. Article

Long, W. C.

2016. A new quantitative model of multiple transitions between discrete stages, applied to the development of crustacean larvae. Fish. Bull. 114:58-66. 
Long, W. C., K. M. Swiney, and R. J. Foy.

2013a. Effects of ocean acidification on the embryos and larvae of red king crab, Paralithodes camtschaticus. Mar. Pollut. Bull. 69:38-47. Article

Long, W. C., K. M. Swiney, C. Harris, H. N. Page, and R. J. Foy. 2013b. Effects of ocean acidification on juvenile red king crab (Paralithodes camtschaticus) and Tanner crab (Chionoecetes bairdi) growth, condition, calcification, and survival. PLoS ONE 8:e60959. Article

Moriyasu, M., and C. Lanteigne.

1998. Embryo development and reproductive cycle in the snow crab, Chionoecetes opilio (Crustacea: Majidae), in the southern Gulf of St. Lawrence, Canada. Can. J. Zool. 76:2040-2048. Article

Nakanishi, T.

1987. Rearing condition of eggs, larvae and post-larvae of king crab. Bull. Japan Sea Reg. Fish. Res. Lab. 37: $57-161$.

NPFMC (North Pacific Fishery Management Council).

2015. Stock assessment and fishery evaluation report for the king and Tanner crab fisheries of the Bering Sea and Aleutian Islands regions: 2015 final crab SAFE, $950 \mathrm{p}$. [Available at website.]

Orensanz, J. M., J. Armstrong, D. Armstrong, and R. Hilborn. 1998. Crustacean resources are vulnerable to serial depletion-the multifaceted decline of crab and shrimp fisheries in the Greater Gulf of Alaska. Rev. Fish Biol. Fish. 8:117-176. Article

Otto, R. S., and P. A. Cummiskey.

1985. Observations on the reproductive biology of golden king crab (Lithodes aequispina) in the Bering Sea and Aleutian Islands. In Proceedings of the International King Crab Symposium; Anchorage, AK, 22-24 January, p. 131-144. Univ. Alaska Fairbanks, Alaska Sea Grant College Program Rep. AK-SG-85-12.

Paul, A. J., and J. M. Paul.

1999. Development of larvae of the golden king crab Lithodes aequispinus (Anomura: Lithodidae) reared at different temperatures. J. Crust. Biol. 19:42-45. Article

2000. Changes in chela heights and carapace lengths in male and female golden king crabs Lithodes aequispinus after molting in the laboratory. Alaska Fish. Res. Bull. 6:70-77.

2001. The reproductive cycle of golden king crab Lithodes aequispinus (Anomura: Lithodidae). J. Shellfish Res. 20:369-372.

$\mathrm{R}$ Development Core Team.

2011. R: a language and environment for statistical computing. R Foundation for Statistical Computing, Vienna, Austria. [Available at website, accessed November 2011.]

Shirley, T. C., and S. Zhou.

1997. Lecithotrophic development of the golden king crab
Lithodes aequispinus (Anomura: Lithodidae). J. Crust. Biol. 17:207-216. Article

Sloan, N.

1985. Life history characteristics of fjord-dwelling golden king crabs Lithodes aequispina. Mar. Ecol. Prog. Ser. 22:219-228.

Somerton, D. A., and R. A. MacIntosh.

1985. Reproductive biology of the female blue king crab Paralithodes platypus near the Pribilof Islands, Alaska. J. Crust. Biol. 5:365-376.

Somerton, D. A., and R. S. Otto.

1986. Distribution and reproductive biology of the golden king crab, Lithodes aequispina, in the eastern Bering Sea. Fish. Bull. 84:571-584.

Stabeno, P. J., D. G. Kachel, N. B. Kachel, and M. E. Sullivan. 2005. Observations from moorings in the Aleutian Passes: temperature, salinity and transport. Fish. Oceanogr. 14:39-54. Article

Stevens, B. G.

2006. Embryo development and morphometry in the blue king crab Paralithodes platypus studied by using image and cluster analysis. J. Shellfish Res. 25:569-576. Article

Stevens, B. G., K. M. Swiney, and L. Buck.

2008. Thermal effects on embryonic development and hatching for blue king crab Paralithodes platypus (Brandt, 1850) held in the laboratory, and a method for predicting dates of hatching. J. Shellfish Res. 27:1255-1263. Article

Swiney, K. M.

2008. Egg extrusion, embryo development, timing and duration of eclosion, and incubation period of primiparous and multiparous Tanner crabs (Chionoecetes bairdi). J. Crust. Biol. 28:334-341. Article

Swiney, K. M., and W. C. Long.

2015. Primiparous red king crab Paralithodes camtschaticus are less fecund than multiparous crab. J. Shellfish Res. 34:493-498. Article

Swiney, K. M., W. C. Long, G. L. Eckert, and G. H. Kruse.

2012. Red king crab, Paralithodes camtschaticus, sizefecundity relationship, and inter-annual and seasonal variability in fecundity. J. Shellfish Res. 31:925-933. Article

Swiney, K. M., W. C. Long, and R. J. Foy.

In press. Effects of ocean acidification on Tanner crab reproduction and early life history, Part I: long-term exposure reduces hatching success and female calcification, and alters embryonic development. ICES J. Mar. Sci.

Webb, J. B., G. L. Eckert, T. C. Shirley, and S. L. Tamone.

2007. Changes in embryonic development and hatching in Chionoecetes opilio (snow crab) with variation in incubation temperature. Biol. Bull. 213:67-75. 